\title{
Predictive Value of Risk Factors for Chronic Idiopathic Thrombocytopenic Purpura in Patients with Acute Type of Disease
}

\author{
Gholamreza Bahoush ${ }^{1}$, Saeede Ghasemi ${ }^{2}$, Pourya Salajegheh ${ }^{3}$, Seyed Mohsen Razavi ${ }^{4}$, Ahmad Bahrami $^{5 *}$ \\ ${ }^{1}$ Hematology and Oncology Ward, Ali-Asghar Children Hospital, Pediatric Departments, Faculty of Medicine, Iran University of \\ Medical Sciences, Tehran, Iran; ${ }^{2}$ Ali-Asghar Children Hospital, Pediatric Departments, Faculty of Medicine, Iran University of \\ Medical Sciences, Tehran, Iran; ${ }^{3}$ Pediatric Hematologist and Oncologist, Pediatric Departments, Faculty of Medicine, Kerman \\ University of Medical Sciences, Tehran, Iran; ${ }^{4}$ Clinic of Hematology and Oncology, Firoozgar Hospital, Iran University of \\ Medical Sciences, Tehran, Iran; ${ }^{5}$ Immunology and Allergy Ward, Ali-Asghar Children Hospital, Pediatric Departments, Faculty \\ of Medicine, Iran University of Medical Sciences, Tehran, Iran
}

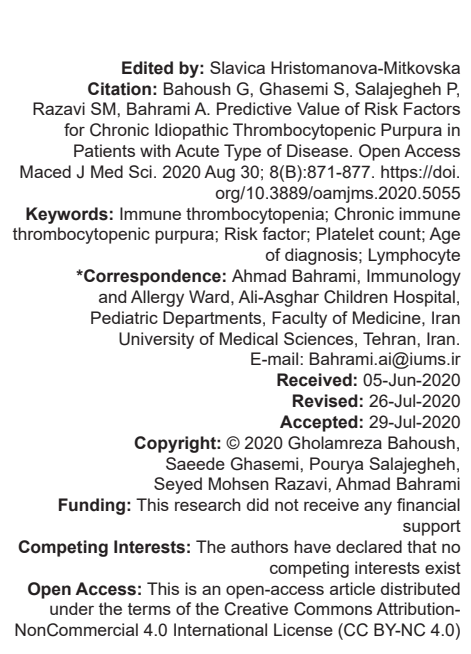

\section{Background}

Platelets play a very important role in homeostasis and their dysfunction (thrombocytopenia) results in hemorrhage, especially in the skin and mucosa. One of the most common chromosomal abnormalities is immune thrombocytopenic purpura (ITP), which has an incidence in children of about $2-5$ cases $/ 100,000$ [1]. ITP is considered to be an acquired, auto-immune disorder, and up to two criteria are needed for the disease: (a) There is only thrombocytopenia and other findings in the complete blood count and peripheral blood smear is normal, (b) The lack of other clinical conditions with similar manifestation such as systemic lupus erythematosus (SLE), antiphospholipid syndrome, and chronic lymphoid leukemia [2]. The above-mentioned patients are considered to be secondary immune thrombocytopenia. Infections with infectious agents such as human immunodeficiency virus, hepatitis c virus, cytomegalovirus, and varicella zoster virus can be associated with such antibodies, leading to secondary ITP [3]. There are also strong reports about the role of Helicobacter pylori infection in the etiology of ITP [4]. It is an autoimmune disease that is associated with a decrease in platelet count, which may occur with a reduction of platelet and platelet life-span. Although the earliest mechanism of autoantibody induction is no known, these autoantibodies are specific and they can be attached to the platelet surface glycoproteins. Specific glycoproteins, including GPIIb/IIla and GPIb/ Ix, can be autoantigen, and due to unknown reason, it 
can stimulate the immune system, leading to antibody production and destruction of platelets [5].

In $75 \%$ of cases, autoantibodies are produced against one or both of the above glycoproteins, and then platelet can be removed from the bloodstream by the phagocytosis or complement-mediated lysis [2]. On the other hand, antibodies binding to megakaryocytes may lead to interruptions in the development of megakaryocytes and release of platelets from megakaryocytes [5].

The disease can be acute or chronic. In acute type, platelet count reaches above $150,000 / \mathrm{mm}^{3}$ after 6 months and no recurrence occurs. In chronic type, low platelet counts persist for more than 6 months [3]. Numerous studies have been done in recent decades on the prevalence, diagnosis, and treatment of ITP [6], [7], [8], but are still open for further investigation and identification of predictive factors. In recent years, therapeutic indications for reducing intravenous immunoglobulin (IVIG) consumption from severe thrombocytopenia $(<20,000)$ have led to treatment only under conditions of bleeding. However, the implementation of these new guidelines, especially in developing countries, has not been welcomed by families of children and therapists. Therefore, the timing of the disease at the onset of its acute form using chronic disease predisposing factors may be helpful. Current treatment of ITPs includes the use of IVIG or steroids, anti-D, and in some cases only the patient is monitored [9]. Old age, female gender, and the onset of disease, silence have been suggested as risk factors for chronic disease [10], [11], [12], but unfortunately it is unclear, which what is the relationship between disease time and the number of bone marrow megakaryocytes. Given the absence of a standard for the chronic type ITP, identifying the risk factors involved in the onset and early diagnosis of the disease is very valuable. Although several studies have examined the risk factors associated with the chronic state of ITP disease [10], [11], [12], early diagnosis of the disease remains a major concern. Therefore, research on finding predictive patterns of various risk factors related to chronic disease is very valuable and will greatly help in diagnosis, appropriate treatment of disease and reduction of the economic burden of society. The aim of this study was to evaluate the predictive value of chronic ITP risk factors in acute ITP patients referred to Ali Asghar and Rasool Akram Hospitals in Tehran, which could lead to a predictive model for identifying chronic cases at the time of diagnosis and reducing treatment costs.

\section{Materials and Methods}

In this prospective cohort study, 65 children under the age of 19 who referred to two hospitals of Ali Asghar and Rasool Akram for ITP were included in the study.

\section{Sample size}

The sample size was calculated based on similar studies. In this study, the sample size was calculated as 150 patients by considering a confidence of $95 \%(\mathrm{Cl}=95 \%)$, the significance level of $<0.05$, and error of 0.08 , but only 65 patients were included in the study within the prescribed period.

\section{Inclusion criteria}

Children under 19 years of age with a diagnosis of acute ITP were included in the study.

\section{Exclusion criteria}

The following criteria were excluded from the study:

1. Patients who did not consent to participate in the study

2. Patients who had thrombocytopenia, leukopenia, and anemia

3. Patients who had drug-induced thrombocytopenia (drugs such as sulfonamides, penicillin, anti-tuberculosis drugs, and anti-inflammatory drugs such as NSAIDs, cimetidine, ranitidine, and anticonvulsants such as diazepam, phenytoin, carbamazepine, valproic acid, antihypertensive drugs, and heart drug such as acetazolamide and captopril)

4. Patients with impaired coagulation tests (PT > 16s and PT $>42$ s)

5. Microangiopathic hemolytic anemia patients

6. Patients with underlying diseases such as congenital platelet function defects (such as syndrome Thrombocytopenia-absent radius syndrome, Bernard-Soulier syndrome, and von Willebrand syndrome)

7. Patients with leukemia, types of viral hepatitis, sepsis, hypersplenism, HIV infection, or vascular collagen diseases such as SLE.

Demographic data, including age, sex, and history of any familial disease, clinical symptoms, manifestations of the disease, and medications taken by patients, were included in the data collection form. Routine laboratory results of hematology, blood smear, and biochemical tests such as CBC, white blood cells (WBC), PMN, ESR, mean platelet volume (MPV), and CRP were evaluated. History of infection and type of infection, including $H$. pylori infection, were also reviewed. Part of the patients' blood samples was also screened for mutations in the FCGR2B gene as well as the type of mutation using the molecular method. Finally, patients returned to the hospital after 6 months and their symptoms, and response to treatment were evaluated using a second peripheral blood smear test. 


\section{Data analysis}

Data were analyzed by SPSS software (version 19). Quantitative data were analyzed using the descriptive program and presented as Mean \pm SD. A Chi-square test was used to compare percentages or frequencies of qualitative data.

The mean of data was compared between the two groups in the chronic and acute groups by independent sample t-test. Multiple logistic regression tests were used to investigate the relationship between different risk factors with chronic disease. In this study, $p<0.05$ was considered statistically significant.

\section{Results}

In this study, 65 patients were included, of which 31 (47.69\%) were male and 34 (52.31\%) were female. Of the 65 patients, $28(43.08 \%)$ had acute ITP and $37(56.92 \%)$ had chronic ITP. Frequency of male and female in acute ITP was equal to $21.54 \%$ and in chronic ITP was $26.15 \%$ and $30.77 \%$, respectively. The findings revealed no significant difference in the frequency of acute and chronic ITP in terms of gender ( $p=0.47$ ). Overall, $69.8 \%$ had a history of infection and $30.2 \%$ had no history of infection. The prevalence of infection in patients with acute and chronic ITP was $35.85 \%$ and $33.96 \%$, respectively. There was no significant difference between the two groups $(p=0.92)$.

The results of risk factors between patients with chronic and acute ITP showed a significant difference in mean primary MPV ( $p=0.009)$, mean age at diagnosis $(p=0.003)$, and lymphocyte count $(p=0.003)$ between the two groups, while no significant difference was found in mean platelet-level risk factors at diagnosis $(p=0.092)$ and primary WBC count $(p=0.722)$.

The mean primary MPV in chronic type ITP patients was significantly higher than that in acute type of ITP ( $p=0.009)$. Mean age at diagnosis was significantly higher in chronic ITP than in acute ITP $(p=0.003)$. The mean lymphocyte count in chronic type of ITP was significantly lower than that in acute type of ITP patients $(p=0.003)$ (Table 1$)$.

Table 1: Comparison of the risk factors for chronic ITP among all enrolled patients

\begin{tabular}{lllllll}
\hline Risk factors & ITP type 2 & $\mathrm{N}$ & Mean & SD & SEM & p-value \\
\hline Initial MPV & Acute & 26 & 10.031 & 0.6085 & 0.1193 & 0.009 \\
\multirow{2}{*}{ Age at diagnosis } & Chronic & 31 & 10.610 & 0.9823 & 0.1764 & \\
& Acute & 28 & 27.38 & 33.483 & 6.328 & 0.003 \\
& Chronic & 37 & 57.81 & 46.304 & 7.612 & \\
PIt at diagnosis & Acute & 28 & 16571.43 & 17733.477 & 3351.312 & 0.092 \\
& Chronic & 35 & 25828.57 & 25130.817 & 4247.883 & \\
Initial WBC & Acute & 28 & 8235.71 & 2071.002 & 391.383 & 0.722 \\
& Chronic & 35 & 8448.57 & 2543.547 & 429.938 & \\
Absolute lymph & Acute & 26 & 5700.54 & 1725.587 & 338.415 & 0.003 \\
count & Chronic & 35 & 4150.03 & 2140.613 & 361.830 & \\
& Chronic & 36 & 22277.77 & 23044.814 & 3840.80 & \\
WBC: White blood cells, ITP: Immune thrombocytopenic purpura, SEM: Standard error of the mean,
\end{tabular}

SD: Standard deviation.
Multiple logistic regression model showed that six factors, including lymphocyte count, age at diagnosis, primary WBC count, platelet level, sex, and MPV, were associated with chronic ITP (Table 2).

Table 2: Multiple logistic regression study of important risk factors for chronicity of ITP

\begin{tabular}{llllllll}
\hline Risk factors & B & SE & Wald & df & Sig. & Exp (B) \\
\hline Step 1 & Absolute lymph count & 2.261 & 0.943 & 5.747 & 1 & 0.017 & 9.591 \\
& Age at diagnosis & -3.699 & 1.403 & 6.948 & 1 & 0.008 & 0.025 \\
& Initial PIt group & -0.713 & 0.823 & 0.751 & 1 & 0.386 & 0.490 \\
Initial WBC & 0.000 & 0.000 & 4.608 & 1 & 0.032 & 1.000 \\
$\quad$ Sex & 0.770 & 0.809 & 0.904 & 1 & 0.342 & 2.159 \\
Initial MPV & 0.712 & 0.570 & 1.559 & 1 & 0.212 & 2.038 \\
$\quad$ Constant & -11.449 & 5.824 & 3.865 & 1 & 0.049 & 0.000 \\
\hline WBC: White blood cells, ITP: Immune thrombocytopenic purpura, SE: Standard error, MPV: Mean platelet \\
volume.
\end{tabular}

There was no significant difference in the frequency of primary platelets $<20,000$ and $\geq 20,000$ in patients with acute and chronic ITP $(p=0.24)$. The results showed that there was no significant difference in the frequency of patients with acute and chronic type ITP based on FCG gene mutation ( $p=0.51$ ). Approximately $27.27 \%$ of patients with acute ITP and $49.09 \%$ of patients with chronic ITP had no mutation in this gene. Frequency of FCG gene mutation in patients with chronic and acute type ITP was $16.36 \%$ and $7.27 \%$, respectively. There was no significant difference in the type of FCG mutation between the two groups $(p=0.78)$ (Figure 1). Frequency of patients based on cutoff age at diagnosis ( $<6$ months and equal to or $>6$ months) showed a significant difference $(p=0.003)$. Approximately, $52.31 \%$ of patients with chronic ITP were more than or equal to 6 months of age, while this frequency was $26.15 \%$ for patients with acute ITP. The frequency of patients with chronic and acute ITP in the age range below 6 months was $4.62 \%$ and $16.92 \%$, respectively.

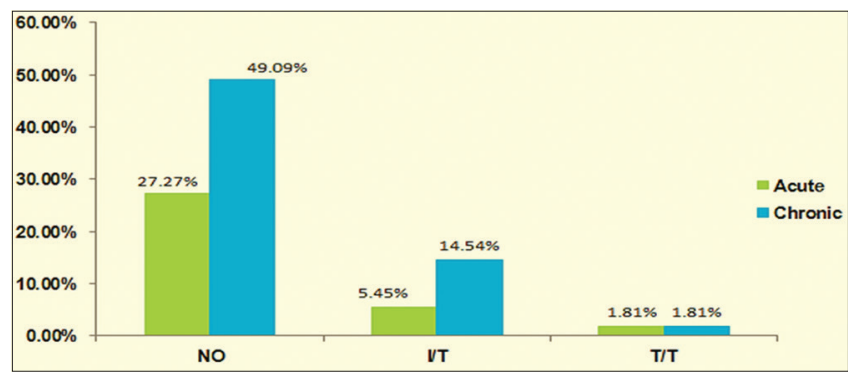

Figure 1: Comparison of frequency of FCG gene mutation among patients with acute and chronic immune thrombocytopenic purpura

The frequency of patients based on the cutoff number of lymphocytes (<4000 and equal or more than 4000) showed significant difference $(p=0.001)$. Approximately $34.43 \%$ of patients with chronic ITP had a lymphocyte count of $<4000$, while this frequency was only $8.20 \%$ for patients with acute ITP. The incidence of patients with chronic and acute ITP with a lymphocyte count of $\geq 4000$ was $22.95 \%$ and $34.43 \%$, respectively.

A comparison of the frequency of patients with chronic and acute ITP based on the primary WBC cutoff ( $<7000$ and more or equal to 7000 ) showed no significant difference in the frequency of chronic and acute ITP based on the primary cutoff WBC ( $p>0.05)$. 
The frequency of chronic and acute ITP patients with $<7000$ WBC was $12.7 \%$ and $20.63 \%$, respectively, while the distribution of chronic and acute ITP with WBC $\geq 7000$ was determined to be $31.75 \%$ and $34.92 \%$, respectively

The minimum cutoff for the diagnosis of chronic cases at the time of diagnosis was 0.31 based on six factors in the model, including initial absolute lymphocyte count, patient age at the time of diagnosis of acute disease, initial WBC count, initial MPV, sex, and initial platelet count using the receiver operating characteristic (ROC) curve (Figure 2).

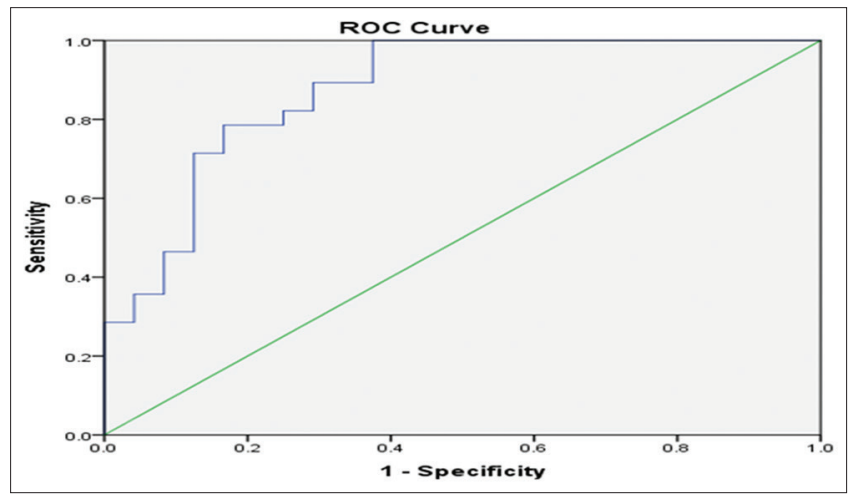

Figure 2: Calculation of cutoff rate for risk factors

Further analysis of the association of these risk factors in comparison with the gold standard showed that the diagnostic sensitivity of these risk factors for chronic ITP was $73.08 \%$, and their specificity was $88.57 \%$, indicating the importance and high predictive power of these risk factors (Figure 3 ).

\begin{tabular}{|c|c|c|c|c|}
\hline \multicolumn{5}{|c|}{ Cross table } \\
\hline \multirow{4}{*}{ Test1 } & \multirow[b]{2}{*}{+} & $\begin{array}{c}\text { Gold standard } \\
+\end{array}$ & & \multirow[b]{2}{*}{23} \\
\hline & & 19 & 4 & \\
\hline & \multirow[t]{2}{*}{ - } & 7 & 31 & 38 \\
\hline & & 26 & 35 & Total $=61$ \\
\hline & \multicolumn{4}{|c|}{ Sensitivity $=73.08 \%(95 \%$ Cr: $53.92-86.3)$} \\
\hline & \multicolumn{4}{|c|}{ Specificity $=88.57 \%(95 \% \mathrm{Cl}: 74.05-95.46)$} \\
\hline & \multicolumn{4}{|c|}{$P P V=82.61 \%(95 \%$ Cl: $62.86-93.02)$} \\
\hline & \multicolumn{4}{|c|}{$\mathrm{NPV}=81.58 \%(95 \% \mathrm{Cl}: 66.58-90.78)$} \\
\hline & \multicolumn{4}{|c|}{ Accuracy $=81.97 \%(95 \%$ Cl: $70.53-89.62)$} \\
\hline & \multicolumn{4}{|c|}{$\mathrm{LR}+=6.394(95 \% \mathrm{Cl}: 2.47-16.556)$} \\
\hline & \multicolumn{4}{|c|}{$L R-=0.304(95 \%$ Cl: $0.16-0.579)$} \\
\hline
\end{tabular}

Figure 3: Comparison of sensitivity and specificity of risk factors for chronic immune thrombocytopenic purpura

\section{Discussion}

The aim of this study was to investigate the predictive value of risk factors for chronic ITP in patients with acute disease. For the first time, we have come up with a model that is able to detect about $90 \%$ of earlystage ITP chronic conditions. The relationship between various factors such as age of diagnosis, sex, history, and type of infection, mutation and its type in FCG gene, platelet count, WBC count, MPV level, and lymphocyte count with chronic ITP was evaluated in patients with acute disease.

Multiple logistic regressions showed that three factors, including absolute lymphocyte count, age at diagnosis and WBC count were independently associated with the chronic condition of ITP. The ROC diagram showed that if the cutoff result of this risk factor was $>0.31$, these risk factors could be used to diagnose or predict chronic status of the disease. Further analysis of the association of these risk factors in comparison with the gold standard demonstrated that their diagnostic sensitivity and specificity for chronic ITP were $73.08 \%$ and $88.57 \%$, respectively, indicating the importance and high predictive power of these risk factors. This model can identify nearly $90 \%$ of those who will become chronic. Thus, for the first time, a proposed model can detect the chronic state of the disease by measuring and evaluating these factors based on the cutoff.

In this study, no significant relationship was found between FCG gene mutation and its type with chronic disease. Furthermore, no relationship was found between the history of infection and the type of infection with the onset of chronic disease, while some previous studies have emphasized on the association of FCG mutation and infection, especially $H$. pylori infection with chronic disease. In a study by Bruin et al., the association of FCG gene mutation and infection history with chronic ITP in 60 pediatric patients showed that the I/T genotype of the FCG gene and history of previous infection was also significantly associated with the development of chronic ITP in children [13]. In another study, Foster et al. [14] examined the association of FCG gene mutations with chronic ITP in 37 children, which the results showed a significant relationship of FCG gene mutations (FCGR3A and FCGR3B) with incidence of chronic condition.

In a recent study, Pavkovic et al. examined the association of FCG gene mutation (FCGR2A) with chronic ITP expression in 120 adult ITP patients, where a significant association between polymorphism in this gene and chronic ITP was observed. However, these researchers recommended conducting a study with a larger sample size [15]. On the other hand, our study there did not demonstrate a relationship of the type of FCG genotype and the history of infection with chronic disease. Given that the FCG mutation study was performed for the first time in a diagnostic center in Iran, there is a possibility of error in the results of the mutation and may be one of the reasons for the lack of significant association between FCG mutation and chronic disease in Iran. On the other hand, a small sample size of chronic patients may be another possible reason for this lack of association. To this end, a larger sample size study is needed to investigate the 
association between FCG mutation or polymorphism and the occurrence of chronic ITP.

In a study by El Alfy et al., the diagnostic value of various predictive factors for the diagnosis of chronic ITP was investigated; their results indicated that $65 \%$ of patients had acute ITP and $34.9 \%$ had chronic ITP [11]. Their results also showed that patients with chronic disease had a significantly higher mean age at the time of diagnosis compared with the acute state, which is consistent with our findings.

In our study, the mean age of diagnosis was significantly higher in chronic patients than in patients with acute disease. They also showed that girls over the age of 10 years were significantly more susceptible to chronicity than boys. On the other hand, the number of primary platelets more than $10^{9} \times 20 / \mathrm{L}$ was considered as a risk factor for chronic disease. In our study, gender and the number of primary platelets were considered as one of the major risk factors for chronic disease, which is in line with these results. Therefore, according to research by these researchers, gender, age above 10 years, and the primary platelet count was considered as important risk factors for chronic ITP [11].

In another study, Ahmed et al. reported the association of the absolute number of leukocytes and blood lymphocytes in our disease prediction [16], which is consistent with the findings of our study. In 2014, Dr. Bahosh et al. also confirmed the association of the absolute number of blood lymphocytes at the time of diagnosis of acute type of disease with its time [17].

In a meta-analysis study by Heitink-Pollé et al., 54 articles on the diagnostic factors of chronic ITP were reviewed and analyzed [10]. Their results showed that various factors such as female gender, older age ( $\geq 11$ years), no history of infection or vaccination, increased platelet count $(>20,000$ units $/ \mu \mathrm{l})$, presence of antinuclear antibodies, and history of treatment with methylprednisolone and IVIG are significantly associated with chronic condition of disease. Children with mucosal hemorrhage were also significantly less susceptible to chronic ITP at the time of diagnosis or treatment with IVIG alone. The results of this study were largely consistent with the findings of our study. Similarly, sex, age, and platelet counts were considered as important risk factors for chronic disease in our study. In another study, Makis et al. examined the relationship of different risk factors with the development of chronic ITP in 57 children [12]. Multiple logistic regression models were used to investigate the association of age, sex, history of infection, type of bleeding, duration of symptoms, platelet count at diagnosis, treatment process, and immunological markers with chronic ITP.

Their results demonstrated that children older than 10 years of age, progressive onset of disease, platelet count above 10,000/ $/$ l, and abnormal immunological markers were highly associated with chronic ITP
There was no association between history of infection and spontaneous bleeding with chronic ITP. Therefore, the researchers suggested that several factors may have contributed to the chronicity of ITP, which warrants further investigation. The results of mentioned study were largely consistent with the findings of our study. Zeller et al. assessed the relationship between different risk factors for the diagnosis of chronic disease in 506 children with ITP [18]. The results of their study using multiple logistic regression model demonstrated that age (0-7 years), gender (girls), and platelet counts are the most important risk factors associated with chronic disease, which are in line with the results of our study.

Jung et al. investigated the association of a variety of clinical and laboratory risk factors in the development of chronic disease in 64 children with ITP [19]. Their results showed that age, the previous history of infection and sudden onset of symptoms are the most important risk factors associated with chronic ITP. Their results also revealed that among patients receiving IVIG, children with a platelet count $<45,000 \mu \mathrm{l}$ after 1 month of receiving IVIG had a significantly higher prevalence of chronic ITP than children with platelets greater than or equal to IVIG.

Another study by $\mathrm{Xu}$ et al. evaluated the relationship between different risk factors and the incidence of chronic disease in 114 children with ITP [20]. Their results using multiple logistic regression model showed that age and number of post-operative platelets are significantly associated with chronic disease.

Coccia et al. investigated the relationship between different clinical and laboratory risk factors and the incidence of chronic disease in children with ITP [21]. Their findings revealed that $20 \%$ of patients had chronic disease. After further investigation using multiple logistic regression model, platelet count was an important diagnostic factor for chronic ITP, which is in line with our results.

In an old study, Robb et al. examined the relationship between various risk factors in the diagnosis of chronic ITP in 289 pediatric patients [22]. About 18\% of patients had a chronic condition. Multiple logistic regressions were used to investigate the relationship of age, sex, season of onset, recent history of viral disease, duration of symptoms, and manifestations with chronic ITP. Their results showed that platelet counts and a history of disease symptoms longer than 2 weeks were considered as important risk factors for chronic ITP. They noted that abnormal platelet count after 28 days of diagnosis is an important diagnostic criterion for chronic disease. This study is in line with the results of our study. In the present study, platelet count at the time of diagnosis was considered as an important diagnostic criterion for chronic disease.

Limitations of the study were the lack of parent collaboration, as well as the lack of easy access to the children with ITP during the study period. 


\section{Conclusions}

Given the high diagnostic value and power (approximately 90\%) and low cost of examining these risk factors in comparison to genetic testing, this is a very economically valuable issue. On the other hand, identifying or diagnosing chronic patients using these risk factors does not require costly IVIG treatment protocols, and other low-cost therapies may replace IVIG for these patients. According to the results of this study, for the first time in Iran, six risk factors, including absolute number of lymphocytes, age at diagnosis, sex, MPV level, platelet level at time of diagnosis, and primary WBC count were considered as the most important risk factors affecting the incidence of chronic ITP.

According to the results of this study, it is recommended to perform this suggested pattern for the identification of chronic ITP before IVIG therapy. However, to introduce this pattern in different treatment centers, it is worthwhile to conduct a study with a larger sample size at several treatment centers for confirmation. It is a worthwhile effort to do further research with a larger sample size for investigating the association of FCG polymorphism with the occurrence of chronic ITP.

\section{Authors' Contributions}

HJJ conducted the study, analyzed the data, and wrote the paper, supervised, and reviewed the manuscript. All authors read and approved the final manuscript.

\section{Availability of Data and Materials}

The datasets used and/or analyzed during the current study are available from the corresponding author on reasonable request.

\section{Ethics Approval and Consent to Participate}

The children participated in the study with full parental consent, ensuring that all children's information was kept confidential. The project was initially approved by the Ethics Committee of Iran University of Medical
Sciences with code IR.IUMS.FDM.REC. 1396.29265

Patients were allowed to leave the study at any time, because of adverse effects or risks.

\section{References}

1. Engert A, Balduini C, Brand A, Coiffier B, Cordonnier C Döhner $\mathrm{H}$, et al.The european hematology association roadmap for european hematology research: A consensus document. Haematologica. 2016;101(2):115-208. https://doi.org/10.3324/ haematol.2015.136739

PMid:26819058

2. Rahiminejad MS, Sadeghi MM, Mohammadinejad $\mathrm{P}$, Sadeghi B, Abolhassani H, Firoozabadi MM, et al. Evaluation of humoral immune function in patients with chronic idiopathic thrombocytopenic purpura. Iran J Allergy Asthma Immunol. 2013;12(1):50-6.

PMid:23454778

3. Cines DB, Liebman H, Stasi R. Pathobiology of secondary immune thrombocytopenia. Semin Hematol. 2009;46 Suppl 2:S2-145. https://doi.org/10.1053/j.seminhematol.2008.12.005 PMid:19245930

4. Keihani M, Shafaeian B, Ahmadi J, Atarchi Z. The report of 28 Cases of thrombotic thrombocytopenic purpura and the risk factors and prognosis in the treatment. Tehran Univ Med J. 2000;58(4):72-8.

5. Gernsheimer T. Chronic idiopathic thrombocytopenic purpura: Mechanisms of pathogenesis. Oncologist. 2009;14(1):12-21. https://doi.org/10.1634/theoncologist.2008-0132 PMid:19144680

6. Kayal L, Jayachandran S, Singh K. Idiopathic thrombocytopenic purpura. Contemp Clin Dent. 2014;5(3):410-4. https://doi. org/10.4103/0976-237x.137976

PMid:25191085

7. Lambert MP, Gernsheimer TB. Clinical updates in adult immune thrombocytopenia. Blood. 2017;129(21):2829-35. https://doi. org/10.1182/blood-2017-03-754119 PMid:28416506

8. Khan AM, Mydra $H$, Nevarez A. Clinical practice updates in the management of immune thrombocytopenia. $\mathrm{P} T$. 2017;42(12):756-63.

PMid:29234214

9. Longhurst HJ, O'Grady C, Evans G, De Lord C, Hughes A Cavenagh $\mathrm{J}$, et al. Anti-D immunoglobulin treatment for thrombocytopenia associated with primary antibody deficiency. $J$ Clin Pathol. 2002;55(1):64-6. https://doi.org/10.1136/jcp.55.1.64 PMid:11825928

10. Heitink-Pollé KM, Nijsten J, Boonacker CW, de Haas M, Bruin MC. Clinical and laboratory predictors of chronic immune thrombocytopenia in children: A systematic review and meta-analysis. Blood. 2014;124(22):3295-307. https://doi. org/10.1182/blood-2014-04-570127 PMid:25305206

11. EIAlfy M, Farid S, Maksoud AA. Predictors of chronic idiopathic thrombocytopenic purpura. Pediatr Blood Cancer. 2010;54(7):959-62. https://doi.org/10.1002/pbc.22481 PMid:20405514

12. Makis A, Gkoutsias A, Palianopoulos T, Pappa E, Papapetrou E, Tsaousi $\mathrm{C}$, et al. Prognostic factors for immune thrombocytopenia outcome in greek children: A retrospective single-centered 
analysis. Adv Hematol. 2017;2017:7878605. https://doi. org/10.1155/2017/7878605

PMid:29362564

13. Bruin $M$, Bierings $M$, Uiterwaal $C$, Révész $T$, Bode $L$, Wiesman ME, et al. Platelet count, previous infection and FCGR2B genotype predict development of chronic disease in newly diagnosed idiopathic thrombocytopenia in childhood: Results of a prospective study. Br J Haematol. 2004;127(5):5617. https://doi.org/10.1111/j.1365-2141.2004.05235.x PMid:15566359

14. Foster CB, Zhu S, Erichsen HC, Lehrnbecher T, Hart ES, Choi E, et al. Polymorphisms in inflammatory cytokines and Fcgamma receptors in childhood chronic immune thrombocytopenic purpura: A pilot study. Br J Haematol. 2001;113(3):596-9. https:// doi.org/10.1046/j.1365-2141.2001.02807.x PMid:11380443

15. Pavkovic M, Petlichkovski A, Karanfilski O, Cevreska L, Stojanovic A. FC gamma receptor polymorphisms in patients with immune thrombocytopenia. Hematology. 2018;23(3):1638. https://doi.org/10.1080/10245332.2017.1377902 PMid:28942727

16. Ahmed I, Rajpurkar M, Thomas R, Chitlur M. Initial lymphocyte count and the development of persistent/chronic immune thrombocytopenic purpura. Pediatr Blood Cancer. 2010:55(3):508-11. https://doi.org/10.1002/pbc.22570 PMid:20658623

17. Bahoush G, Motamedi D, Vossough P. Initial lymphocyte count in patients with acute immune thrombocytopenic purpura: Can it predict persistence of the disease? Minerva Pediatr. 2014 PMid:25502733

18. ZellerB, Rajantie J, Hedlund-Treutiger I, Tedgård U, Wesenberg F, Jonsson OG, et al. Childhood idiopathic thrombocytopenic purpura in the Nordic countries: Epidemiology and predictors of chronic disease. Acta Paediatr. 2005;94(2):178-84. https://doi. org/10.1111/j.1651-2227.2005.tb01887.x

PMid: 15981751

19. Jung JY, Rum AO, Kim JK, Park M. Clinical course and prognostic factors of childhood immune thrombocytopenia: Single center experience of 10 years. Korean J Pediatr. 2016;59(8):335-40. https://doi.org/10.3345/kjp.2016.59.8.335

PMid:27610182

20. Xu T, Li N, Jin F, Wu K, Ye Z. Predictive factors of idiopathic thrombocytopenic purpura and long-term survival in Chinese adults undergoing laparoscopic splenectomy. Surg Laparosc Endosc Percutan Tech. 2016;26(5):397-400. https://doi. org/10.1097/sle.0000000000000314

\section{PMid:27749769}

21. Coccia P, Ruggiero A, Attinà G, Cerchiara G, Battista $A$, Arena R. Chronic idiopathic thrombocytopenic purpura in children: Predictive factors and outcome. Cent Eur J Med. 2012;7(4):5258. https://doi.org/10.2478/s11536-012-0011-2

22. Robb LG, Tiedeman K. Idiopathic thrombocytopenic purpura: Predictors of chronic disease. Arch Dis Child. 1990;65(5):502-6. https://doi.org/10.1136/adc.65.5.502

PMid:2357088 